\title{
Steering Acoustically Propelled Nanowire Motors toward Cells in a Biologically Compatible Environment Using Magnetic Fields
}

Suzanne Ahmed, ${ }^{\dagger}$ Wei Wang, ${ }^{\dagger}$ Lamar O. Mair, ${ }^{\#, \nabla}$ Robert D. Fraleigh, ${ }^{\ddagger}$ Sixing Li, ${ }^{\S, \perp}$ Luz Angelica Castro, ${ }^{\text {II }}$ Mauricio Hoyos, "T Tony Jun Huang," and Thomas E. Mallouk*,†,

\author{
${ }^{\dagger}$ Department of Chemistry, ${ }^{\ddagger}$ Department of Physics, ${ }^{\S}$ Department of Biochemistry and Molecular Biology, ${ }^{\|}$Department of \\ Engineering Science and Mechanics, and ${ }^{\perp}$ Department of Cell and Developmental Biology Graduate Program, The Pennsylvania \\ State University, University Park, Pennsylvania 16802, United States \\ ${ }^{\#}$ Center for Nanoscale Science and Technology, National Institute of Standard and Technology, 100 Bureau Drive, Stop 6200, \\ Gaithersburg, Maryland 20899, United States \\ ${ }^{\nabla}$ Maryland Nanocenter, University of Maryland, College Park, Maryland 20742, United States \\ ${ }^{\mathbb{I}}$ Laboratoire de Physique et Mécanique des Milieux Hétérogènes, UMR7636 CNRS, UMPC, ESPCI, 10 rue Vauquelin, 75005 Paris, \\ France
}

\section{Supporting Information}

\begin{abstract}
The recent discovery of fuel-free propulsion of nanomotors using acoustic energy has provided a new avenue for using nanomotors in biocompatible media. Crucial to the application of nanomotors in biosensing and biomedical applications is the ability to remotely control and steer them toward targets of interest, such as specific cells and tissues. We demonstrate in vitro magnetic steering of acoustically powered nanorod motors in a biologically compatible environment. Steering was accomplished by incorporating $(40 \pm 5)$
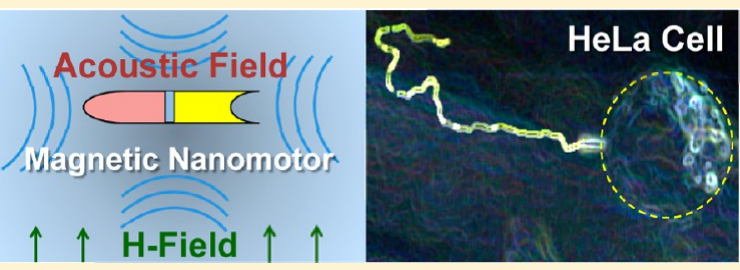
$\mathrm{nm}$ thick nickel stripes into the electrochemically grown nanowires. An external magnetic field of 40-45 mT was used to orient the motors, which were acoustically propelled along their long axes. In the absence of a magnetic field, $(300 \pm 30) \mathrm{nm}$ diameter, $(4.3 \pm 0.2) \mu \mathrm{m}$ long nanowires with $(40 \pm 5) \mathrm{nm}$ thick magnetic stripes exhibit the same self-acoustophoretic behavior, including pattern formation into concentric nanowire circles, aligned spinning chains, and autonomous axial motion, as their non-magnetic counterparts. In a magnetic field, these wires and their paths are oriented as evidenced by their relatively linear trajectories. Coordinated motion of multiple motors and targeting of individual motors toward HeLa cells with micrometer-level precision was demonstrated.
\end{abstract}

\section{INTRODUCTION}

Research into the propulsion and control of nano- and microscale motors has grown steadily over the past decade. $^{1-10}$ Some of the most interesting potential applications of micromotors are in biosensing and biomedicine, but the most widely studied catalytic propulsion mechanism tends to involve toxic fuels or is incompatible with media or high ionic strength. $^{11-16}$ The recent discovery of the propulsion of nanomotors using acoustic energy has provided a new opportunity to explore their behavior in biocompatible media. ${ }^{17,18}$ Self-acoustophoretic nanowire motors are especially attractive because they allow for continuous propulsion that is not limited by the supply of fuel. They are operated in a frequency and power range that is biologically safe. ${ }^{17,19,20}$ The power density used in the experiments described in this paper is $(13 \pm 1) \mathrm{mW} / \mathrm{cm}^{2}$, which is well below the United States Food and Drug Administration (FDA) limit of $740 \mathrm{~mW} / \mathrm{cm}^{2}$ for diagnostic ultrasound. ${ }^{19,20}$ The self-acoustophoretic mechanism also allows one to change the motor speed easily by adjusting the applied power. ${ }^{21-26}$ However, to take full advantage of ultrasonically propelled motors, one must be able to direct and guide their motion toward targets of interest, such as cells and tissues. Here, we demonstrate that remote steering and targeting of acoustically powered motors is possible using externally applied magnetic fields. As in previous reports of magnetically oriented autonomous nanomotors, a magnetic segment was incorporated into the nanowires. ${ }^{27-29} \mathrm{~A}$ ferromagnetic nickel stripe was electrochemically grown between diamagnetic $\mathrm{Au}$ and $\mathrm{Ru}$ segments, as shown in Figure 1 , and it responded to a weak external magnetic field that could be oriented to define the path of the motor in two dimensions.

\section{BEHAVIOR OF NANOMOTORS IN STATIC MAGNETIC FIELDS}

When excited by ultrasonic standing waves near the resonant frequency of the cylindrical cell $(3.77 \pm 0.01 \mathrm{MHz})$, nanowires with $(40 \pm 5) \mathrm{nm}$ thick magnetic stripes exhibited the same behavior as their non-magnetic counterparts, including

Received: October 11, 2013

Revised: December 9, 2013

Published: December 10, 2013 


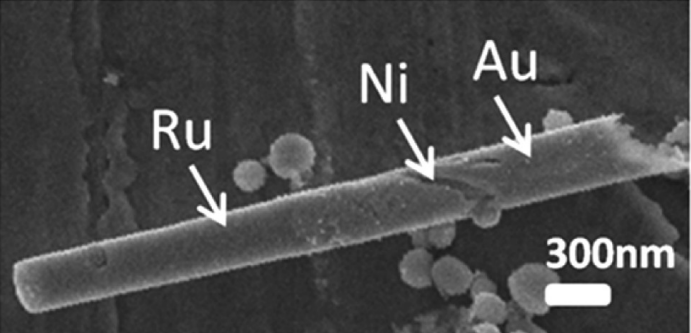

Figure 1. Representative field emission scanning electron microscopy (FE-SEM) image of the $\mathrm{Au}-\mathrm{Ni}-\mathrm{Ru}$ nanowire motors.

levitation to the midpoint of the cell, pattern formation into concentric nanowire circles, alignment into spinning chains, and autonomous axial motion. ${ }^{17}$ The polar alignment of $\mathrm{Au}-$ $\mathrm{Ni}-\mathrm{Ru}$ wires in spinning chains at the acoustic nodal lines, with the ruthenium end leading, was also consistent with earlier observations with non-magnetic $\mathrm{Au}-\mathrm{Ru}$ nanorods. The average speed of the wires did not change with the application of a static magnetic field; speeds of $(26 \pm 12)$ and $(26 \pm 18) \mu \mathrm{m} / \mathrm{s}$ were observed, without and with the field, respectively [the resulting Reynolds number is approximately $(1.0 \pm 0.8) \times$ $\left.10^{-4}\right]$. These values are based on 46 measurements of wires with an applied magnetic field and 120 measurements of wires with no applied field. The positional measurement error was less than $0.3 \mu \mathrm{m}$, and thus, the distribution in speed derives primarily from wire-to-wire speed variation. However, the pattern of movement changed markedly, as shown in Figure 2. In the absence of a magnetic field, wire trajectories followed loops (possibly from slight bending of the wires and asymmetry in the wire end shape) and also contained random turns, but in the magnetic field, they followed straight line trajectories. In the static field (applied parallel to the $y$ axis in Figure 2), the majority of the wires oriented with their long axis within $25^{\circ}$ of the direction perpendicular to the field (the $x$ axis in Figure 2). This is as expected because the thickness of the magnetic stripe of $(40 \pm 5) \mathrm{nm}$ is smaller than its diameter of $(300 \pm 30) \mathrm{nm}$; thus, the easy axis of magnetization should lie in the plane of the stripe. ${ }^{27,30}$ The range of angles observed between the direction of movement and the applied field can be explained by the fact that the Ni stripes in the wires in general were only approximately perpendicular to the wire axis (Figure 1). These static field experiments were conducted at a constant field of
$(40 \pm 1) \mathrm{mT}$ by placing a $\mathrm{Nd}-\mathrm{Fe}-\mathrm{B}$ magnet $(6.60 \pm 0.03) \mathrm{cm}$ from the active area and in the two-dimensional plane of the acoustic cell.

This field strength applied a maximum torque of $(2.0 \pm 0.5)$ $\times 10^{-16} \mathrm{~N}$ m on the nanowires within the cell, as calculated from eq 1

$$
\tau=\mu \times B
$$

where $\tau$ is the torque, $\mu$ is the magnetic dipole moment of the wire $(6.5 \pm 0.5) \times 10^{-15} \mathrm{~A} \cdot \mathrm{m}^{2}$ as determined by ensemble superconducting quantum interference device (SQUID) magnetometry measurements (Figure 3 ), and $B$ is the field strength of the aligning magnet. From this value, the energy required to turn the nanowire through $90^{\circ}$ (i.e., to align the long axis parallel to the field) is approximately $2 \times 10^{-16} \mathrm{~J}$, which is orders of magnitude larger than the thermal energy $k_{\mathrm{B}} T \approx 4 \times 10^{-21} \mathrm{~J}$.

In the low Reynolds number limit, the acoustic propulsion force on the wires is equal to the drag force experienced by the wires. The axial propulsion force was determined from the Stokes drag eq 2 to be $(0.26 \pm 0.05) \mathrm{pN}$ for wires traveling at $25 \mu \mathrm{m} / \mathrm{s}$

$$
F_{\text {drag }}=\frac{2 \pi \eta L}{\ln \left(\frac{L}{R}\right)-0.72} v
$$

where $\eta$ is the dynamic viscosity of water at $293 \mathrm{~K}, L$ is the length of the wire, $R$ is its radius, and $v$ its velocity. ${ }^{31,32}$ In contrast, the axial propulsion force on the nanowires because of the magnetic field was negligible, as evidenced by the fact that there is no change in the speed of the wires before and after the application of the field. To quantify the axial magnetic force, the magnetic field profile was measured as a function of the distance (see Figure S1 of the Supporting Information), and the gradient at the distance $(6.60 \pm 0.03) \mathrm{cm}$ between the sample and the magnet was found to be $(-4.02 \pm 0.02) \mathrm{mT} / \mathrm{cm}$. From this value, the axial magnetic force was calculated to be $(2.6 \pm$ $0.1) \times 10^{-15} \mathrm{~N}$. This is approximately two orders of magnitude smaller than the axial acoustic force.

The movement of acoustically propelled wires in the presence and absence of a magnetic field was quantified using two parameters: the rotational diffusion coefficient and the mean displacement angle of the wires. The rotational diffusion coefficient, which is a measure of the random rotation of the
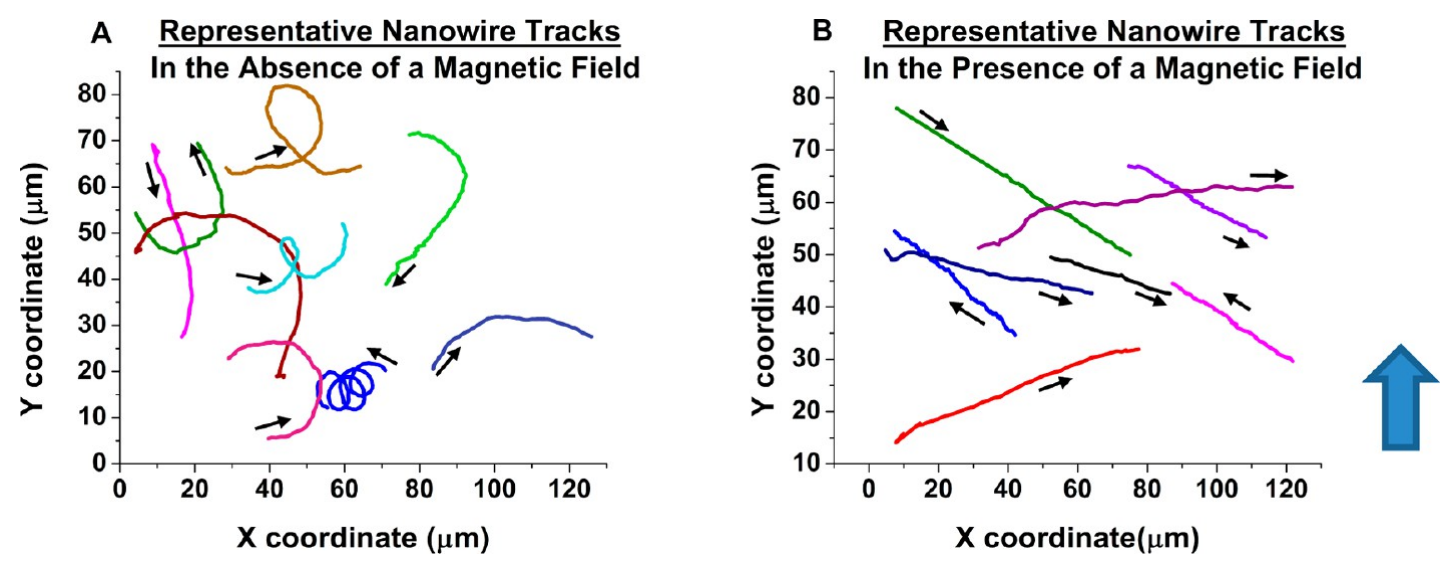

Figure 2. Representative nanowire tracks plotted from the $x$ and $y$ coordinates obtained from tracking nanowires. The bottom left of the screen is defined as the origin. Arrows on the tracks indicate the direction of motion. (A) Representative tracks in the absence and (B) presence of a magnetic field. The field direction is indicated by the large arrow. 

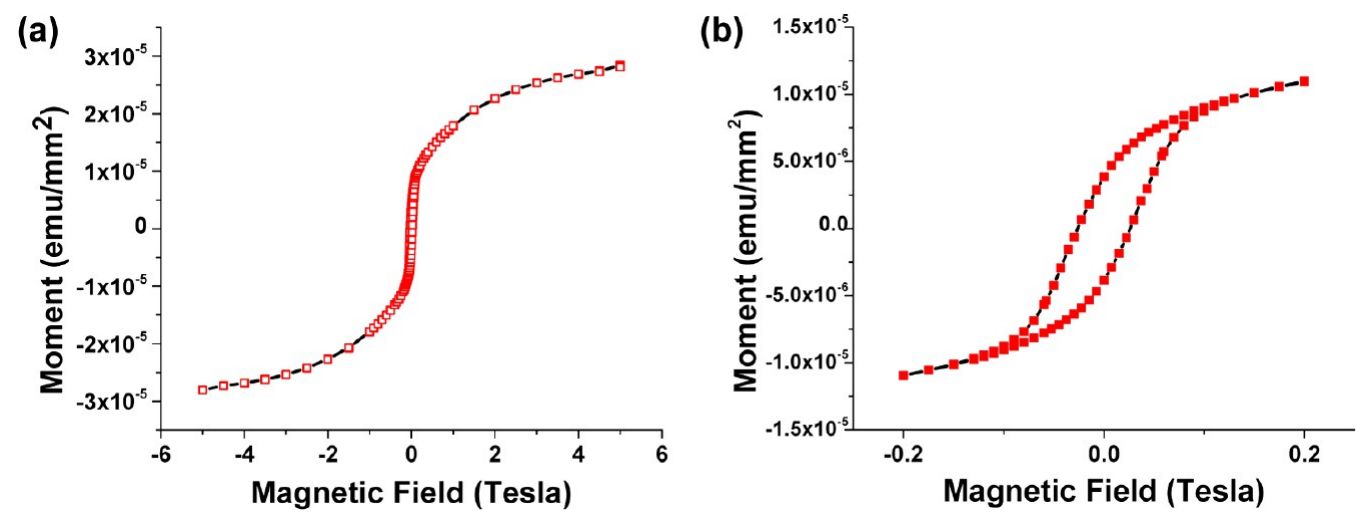

Figure 3. SQUID magnetometry measurements. (A) Hysteresis curve of a $\mathrm{Au}-\mathrm{Ni}-\mathrm{Ru}$ nanowire sample from -5 to $5 \mathrm{~T}$ showing the saturation magnetization used to calculate the dipole moment of the wires. (B) Zoomed in hysteresis curve from -0.25 to $0.25 \mathrm{~T}$ showing the ferromagnetism of the nanowires.

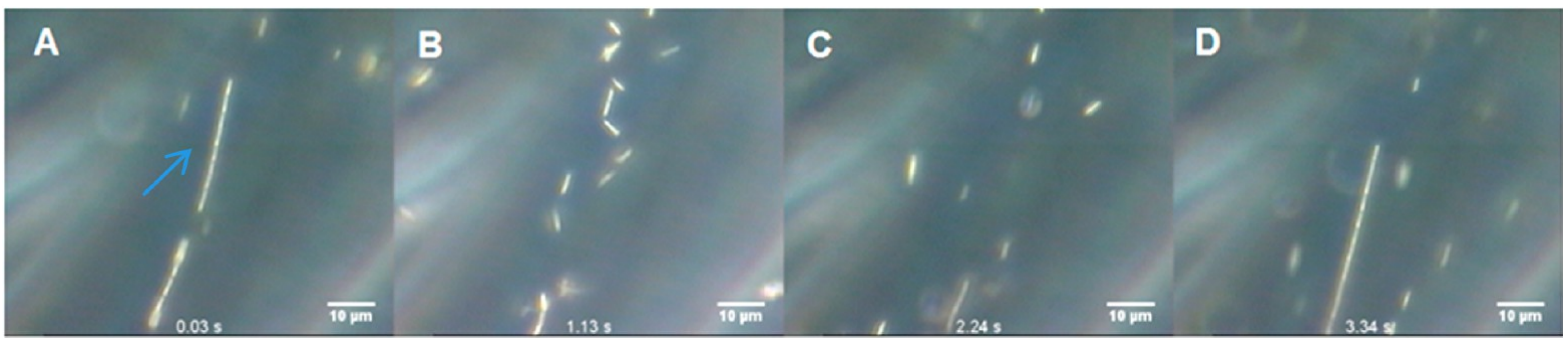

Figure 4. Nanowire motors within an acoustic cell can be confined within acoustic nodal locations, eventually forming patterns, such as spinning chains. (A) Multiple magnetic nanowire motors forming dynamic spinning chains within nodal lines. (B) Upon the application of an oscillating magnetic field, the chain is rapidly disrupted and the nanowire motors are reoriented and are not confined to the nodal lines. (C and D) Upon the removal of the field, the chain reorganizes. Times of frames A-D are indicated (see Video S1 of the Supporting Information). The scale bars represent $10 \mu \mathrm{m}$.

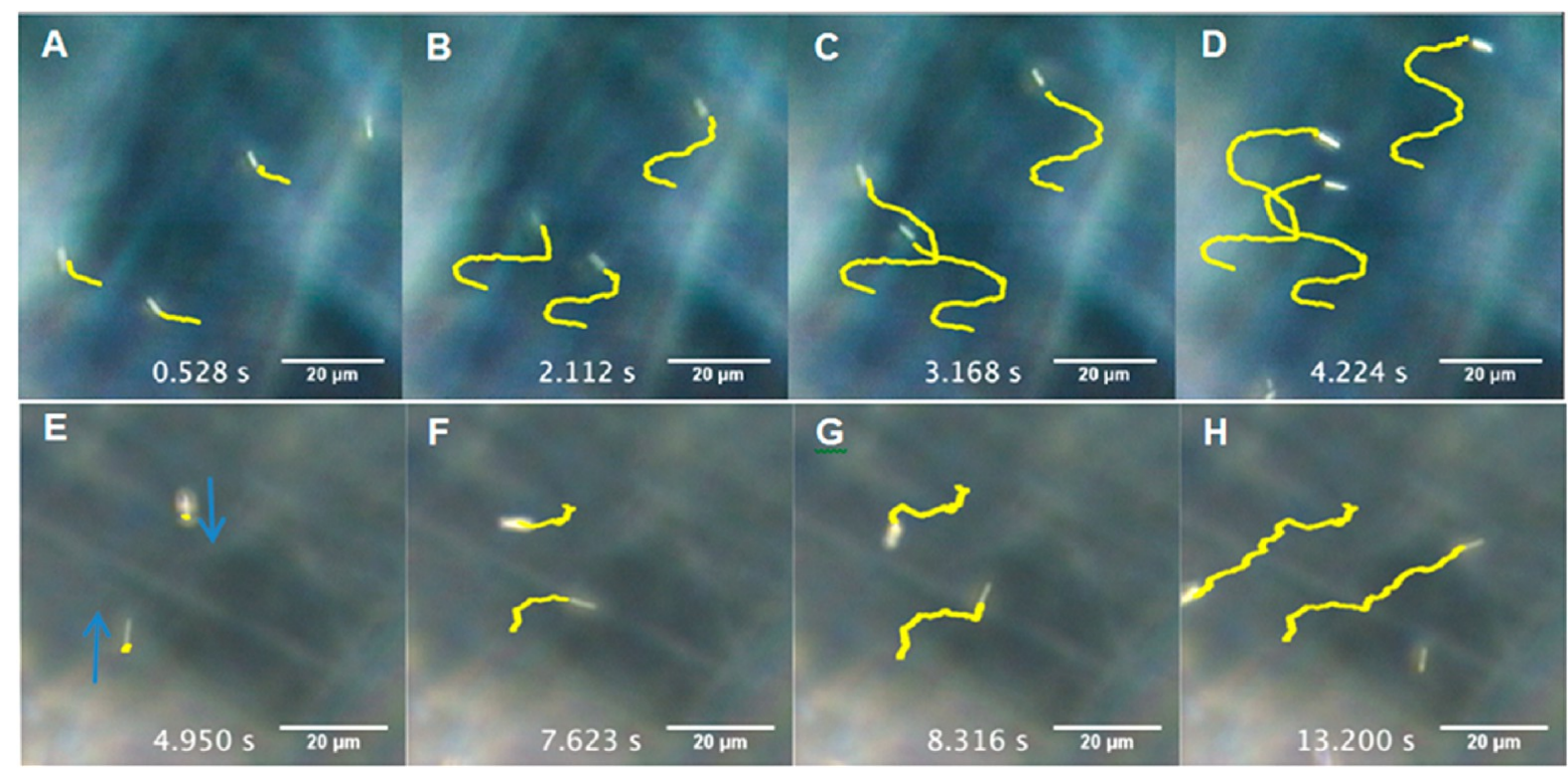

Figure 5. (Top panel) Steered ensemble motion of wires moving at $(25 \pm 5) \mu \mathrm{m} / \mathrm{s}$, showing (A) earlier and (B) later times in the trajectory. Yellow tracking lines are superimposed on the final frame of the video. The wires undergo multiple guided U-turns (see Videos S2 and S3 of the Supporting Information). Times of frames A-D are indicated. (Bottom panel) Autonomous steering of motors moving at $(8 \pm 3) \mu \mathrm{m} / \mathrm{s}$. Times of frames $\mathrm{E}-\mathrm{H}$ are indicated, and arrows show the initial direction of motion in E and consequent guided sharp U-turns (F-H) (see Video S4 of the Supporting Information).

wire axis during propulsion, was determined by tracking the location of the head and tail of the wire over $1 \mathrm{~s}$ for wires undergoing random autonomous motion both before and after the application of the field. ${ }^{33} \mathrm{~A}$ decrease in the rotational diffusion coefficient from $(2.6 \pm 1.1)$ to $(0.5 \pm 0.2)$ degrees $^{2} / \mathrm{s}$ was observed, indicating that the magnetic field suppressed rotational diffusion. The mean displacement angle is the angular deviation in a wire path, which quantifies how straight 

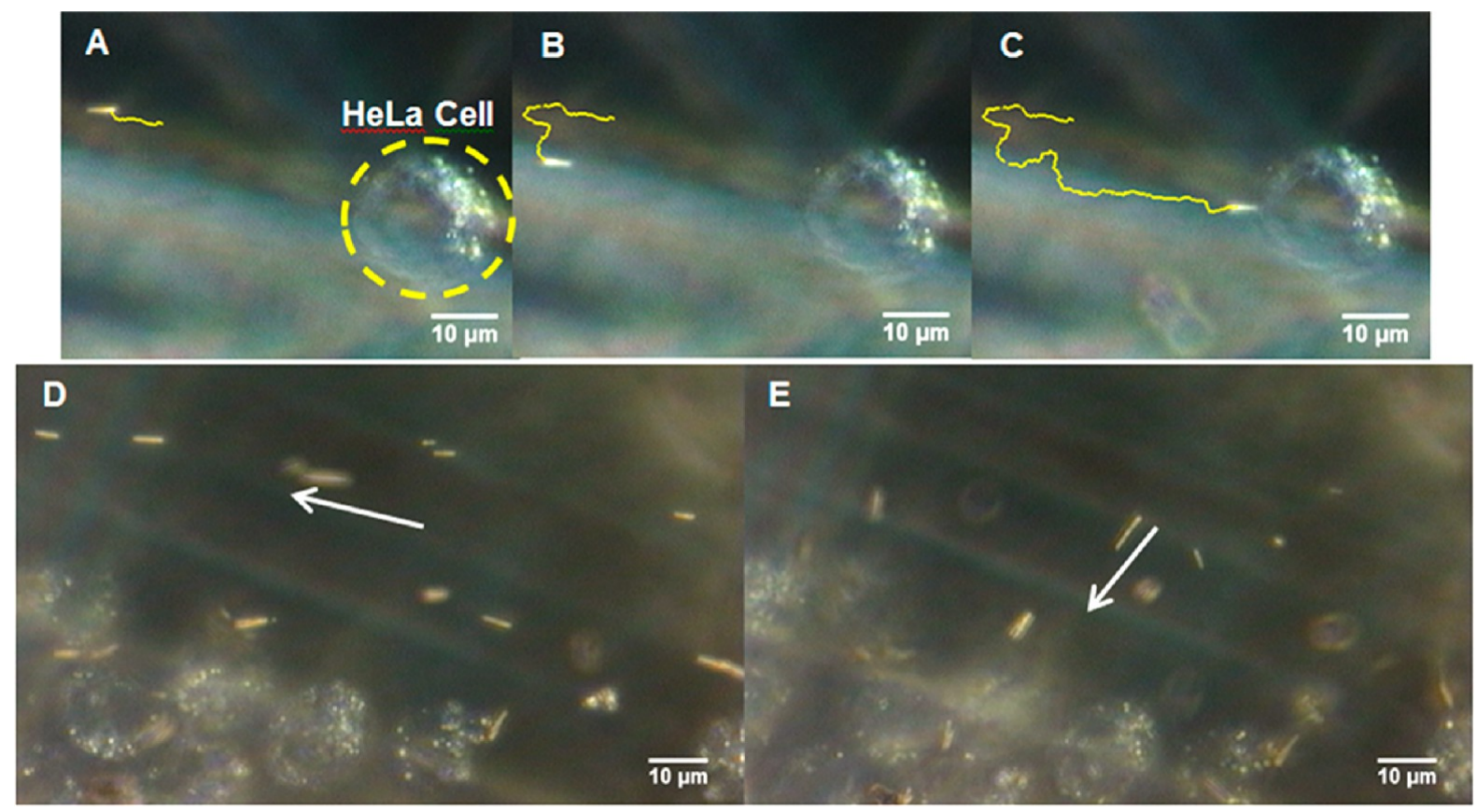

Figure 6. (Top panel) (A-C) Nanomotor targeting toward a HeLa cell. Nanowires were guided to make multiple U-turns to demonstrate control before being steered toward the live cell (see Video S5 of the Supporting Information). (Bottom panel) (D) Nanowires are moving parallel to a group of cells, with their direction indicated by the arrow. (E) When the external field is turned, nanowires are magnetically steered through an approximately $90^{\circ}$ turn toward the cells (see Video S6 of the Supporting Information).

the movement of the wire is. ${ }^{34}$ This angle was measured at $0.2 \mathrm{~s}$ intervals over a minimum of $3 \mathrm{~s}$ for each wire. The mean displacement angle in the absence of the magnetic field was $31.8^{\circ} \pm 9.1^{\circ}$ and decreased to $4.4^{\circ} \pm 1.5^{\circ}$ in the field.

\section{MAGNETIC STEERING}

Using hand-held magnets at a distance of $2.5 \pm 0.1 \mathrm{~cm}$, which apply a $(45 \pm 1) \mathrm{mT}$ field at the sample location [and, hence, a gradient of $(-3.80 \pm 0.01) \mathrm{mT} / \mathrm{cm}$ and an insignificant axial force of $2.4 \times 10^{-15} \mathrm{~N}$ ], it was possible to reversibly disrupt patterns, such as spinning chains that are formed in the acoustic field, by reorienting the wires perpendicular to the chain. The disruption of pattern formation can be seen in Figure 4 and Video S1 of the Supporting Information.

Acoustically propelled motors can travel at axial speeds up to $200 \mu \mathrm{m} / \mathrm{s}^{17}$ Magnetic steering was demonstrated on acoustically propelled nanowire motors moving at axial speeds ranging from $(8 \pm 3)$ to $(170 \pm 10) \mu \mathrm{m} / \mathrm{s}$ (see Figure $\mathrm{S} 2$ of the Supporting Information). This is illustrated by the ensemble motion of wires, which could be directed to move in concert, as shown in Figure 5. The autonomous nature of the acoustically propelled motion was retained when the magnetic field was applied. The top panel of Figure 5 illustrates the magnetic steering of groups of motors that were initially traveling in approximately the same direction and then taken through a series of turns by reorienting the external field. The bottom panel of Figure 5 shows the effect of the field on wires traveling in opposite directions. These wires are also reoriented in the field but in opposite directions and were also made to take multiple sharp U-turns.

\section{CELL TARGETING EXPERIMENTS}

For possible bioanalytical and biomedical applications, it is important to show that acoustically powered nanomotors can be steered in a biocompatible environment. Here, we demonstrate the magnetic steering of nanomotors toward live HeLa cells in an aqueous phosphate buffer. Nanomotors were mixed with HeLa cells and placed into the acoustic cell. Cell viability tests showed no significant degradation in the presence of nanowires after $20 \mathrm{~min}$ of exposure to acoustic excitation at the power levels used in the steering experiments (see the Supporting Information). In Video S5 of the Supporting Information, one nanomotor was selected and guided toward a cell. At the start of the video (see Video S5 of the Supporting Information), to demonstrate directional control, the wire was taken through multiple $180^{\circ}$ turns before guiding it toward the targeted cell. It was possible to steer nanowire motors toward cells that were adjacent in aggregates and, hence, only micrometers apart. Targeting of a single nanowire toward an individual cell and an approximately $90^{\circ}$ turn of a group of nanowires into a group of cells are illustrated in Figure 6. Nanowire targeting toward cells was successfully repeated multiple times to ensure the robustness of the technique.

These experiments have demonstrated that it is possible to suppress random motion and exert relatively fine control over the steering of acoustically propelled nanomotors using a weak external magnetic field. Both the propulsion and steering of these motors can be carried out in biocompatible buffers, as evidenced by steering of motors toward live cells in these media.

\section{MATERIALS AND METHODS}

Precision of Measurements. Uncertainties in measurements were derived primarily from experiment-to-experiment distributions. Measurement error for each of the measured values was less than 5\% of the reported uncertainty, and all representations of uncertainty are given as 1 standard deviation.

Nanowire Growth and Characterization. Bimetallic and trimetallic nanowires were grown electrochemically using commercial anodic aluminum oxide (AAO) membranes (Whatman, Inc.; with nominal pore diameter of $200 \mathrm{~nm}$ ) as templates. Segmented gold- 
nickel-ruthenium wires were made by sequentially changing the plating solution within an electrochemical cell. A two-electrode electrochemical cell was used for the plating of silver, nickel, and gold. ${ }^{35}$ A silver layer evaporated onto the AAO membrane served as the cathode, and a platinum wire served as the anode. Ruthenium was plated in a three-electrode cell with an additional $\mathrm{Ag} / \mathrm{AgCl}$ electrode serving as the reference electrode. A thin gold adhesion layer was deposited between the nickel and ruthenium segments to prevent nanowire fragmentation during release. Gold and ruthenium deposition was conducted under conditions identical to those used previously to yield wires with similar morphological and shape asymmetry. ${ }^{17}$ Nickel was deposited at a constant current density of 0.7 $\mathrm{mA} / \mathrm{cm}^{2}$ for $3 \mathrm{~min}$.

The lengths of the nickel segments were $(40 \pm 5) \mathrm{nm}$, smaller than a single magnetic domain, which is typically $\approx 150 \mathrm{~nm}$, and also smaller than the diameter of the nanorod. This ensured that the easy axis and, hence, direction of magnetization were approximately perpendicular to the nanowire long axis. ${ }^{27,30,36}$ In this arrangement, the motors can be steered with a magnetic field in the plane of the acoustic cell; the field applies substantial torque but minimal axial force to the nanowire.

The magnetic properties of the nanowires were characterized using SQUID magnetometry. The magnetic susceptibility of a $7 \mathrm{~mm}^{2}$ portion of the template with embedded wires was measured at $5 \mathrm{~K}$ from 5 to $-5 \mathrm{~T}$. A background measurement of a control sample containing gold-ruthenium wires grown under identical conditions but without the nickel segment was made for subtraction from the sample measurement. The magnetic dipole moment per wire $(\mu)$ was determined by dividing the total saturation magnetization of the sample by the number of wires contained within it and was determined to be approximately $(6.5 \pm 0.5) \times 10^{-15} \mathrm{~A} \mathrm{~m}^{2}$. This value was used to determine the torque and force on the wires in the applied magnetic fields (eq 1).

Acoustic Propulsion Experiments. The cylindrical acoustic cell with a height of $(180 \pm 10) \mu \mathrm{m}$ and a diameter of $(5.0 \pm 0.1) \mathrm{mm}$ has been described in an earlier work and was used in all experiments. ${ }^{17}$ The solution in the cell was excited by a piezoelectric disc transducer $(1 \mathrm{~mm}$ thick) affixed to the center of a stainless-steel plate $(4.2 \mathrm{~cm} \times$ $4.2 \mathrm{~cm} \times 1 \mathrm{~mm}$ ). Kapton tape was applied to the opposite side of the steel plate, and a hole punched in the center of the tape defined the cell. A glass microscope coverslip was placed on the cell during the experiments as a reflector to set up a standing wave. The resonant frequency of the cell center was $(3.77 \pm 0.01) \mathrm{MHz}$. Experiments were conducted on wires in the levitation plane at the midpoint of the cell at frequencies close to the resonant frequency. The voltage had a peakto-peak value of $10 \mathrm{~V}$ and was applied using a waveform generator.

The behavior of the acoustically propelled nanowire motors in the presence of a static magnetic field was studied and compared to their behavior in the absence of a magnetic field. A cylindrical $\mathrm{Nd}-\mathrm{Fe}-\mathrm{B}$ magnet $(2.50 \pm 0.01) \mathrm{cm}$ in diameter and $(7.60 \pm 0.01) \mathrm{cm}$ in length was held in the plane of the cell at a fixed distance to maintain a constant field strength at the sample. The strength of the applied magnetic field was measured using a digital direct current (DC) gaussmeter. The average speed for 46 and 120 wires was determined with the magnetic field on and off, respectively. For steering experiments, hand-held magnets were used. The external magnetic field was applied using six rectangular $\mathrm{Nd}-\mathrm{Fe}-\mathrm{B}$ magnets $(2.5 \times 2.5 \times$ $0.625 \mathrm{~cm}$ ) in the plane of the cell, at a distance of $2.5 \pm 0.1 \mathrm{~cm}$ from the wires. The strength of the applied magnetic field was measured using a digital DC gaussmeter. A schematic of the setup can be seen in the Supporting Information.

Nanowire motion was tracked using the open access program Video Spot Tracker (http://cismm.cs.unc.edu/downloads/?dl_cat=3) in both the presence and absence of a magnetic field. Videos of nanomotor motion were taken at $500 \times$ magnification at a frame rate of $30 \mathrm{~s}^{-1}$.

For cell targeting experiments, living human cervical cancer cells (HeLa cells) were used. The cells were used within 1 day of culture and stored refrigerated in a phosphate buffer solution that is $0.1 \mathrm{M}$ phosphate and $0.15 \mathrm{M} \mathrm{NaCl}$ at a $\mathrm{pH}$ of 7.2. Details of cell growth and cell viability tests can be found in the Supporting Information.

\section{ASSOCIATED CONTENT}

\section{Supporting Information}

Videos of nanowire motion, details of magnetic force calculations, frame sequence illustrating steering of fast nanowires, schematic of the acoustic cell, and HeLa cell viability experiments. This material is available free of charge via the Internet at http://pubs.acs.org.

\section{AUTHOR INFORMATION}

\section{Corresponding Author}

*E-mail: tem5@psu.edu.

\section{Notes}

The authors declare no competing financial interest.

\section{ACKNOWLEDGMENTS}

We thank Prof. Nitin Samarth for helpful discussions and use of the SQUID magnetometer. This work was supported by the National Science Foundation under MRSEC Grant DMR0802404. Analytical instrumentation used in this work was supported by the Pennsylvania State University Materials Research Institute Nanofabrication Laboratory under National Science Foundation Cooperative Agreement ECS-0335765. The tracking software Video Spot Tracker was developed at the CISMM at the University of North Carolina at Chapel Hill, supported by the National Institute of Biomedical Imaging and Bioengineering (NIBIB) of the National Institutes of Health (NIH) (NIH 5-P41-RR02170). Lamar O. Mair acknowledges support under the Cooperative Research Agreement between the University of Maryland and the National Institute of Standards and Technology Center for Nanoscale Science and Technology, Award 70ANB10H193, through the University of Maryland. Certain commercial equipment, instruments, or materials are identified in this paper to foster understanding. Such identification does not imply recommendation or endorsement by the National Institute of Standards and Technology, nor does it imply that the materials or equipment identified are necessarily the best available for the purpose.

\section{REFERENCES}

(1) Mallouk, T.; Sen, A. Powering nanorobots. Sci. Am. 2009, 300, $72-77$.

(2) Ozin, G. A.; Manners, I.; Fournier-Bidoz, S.; Arsenault, A. Dream nanomachines. Adv. Mater. 2005, 17, 3011-3018.

(3) Fournier-Bidoz, S.; Arsenault, A. C.; Manners, I.; Ozin, G. A. Synthetic self-propelled nanorotors. Chem. Commun. 2005, 441-443.

(4) Mirkovic, T.; Zacharia, N. S.; Scholes, G. D.; Ozin, G. A. Nanolocomotion-Catalytic nanomotors and nanorotors. Small 2010, $6,159-167$.

(5) Gibbs, J. G.; Zhao, Y. P. Design and characterization of rotational multicomponent catalytic nanomotors. Small 2009, 5, 2304-2308.

(6) Gibbs, J.; Zhao, Y. Catalytic nanomotors: Fabrication, mechanism, and applications. Front. Mater. Sci. 2011, 5, 25-39.

(7) Sengupta, S.; Ibele, M. E.; Sen, A. Fantastic voyage: Designing self-powered nanorobots. Angew. Chem., Int. Ed. 2012, 51, 8434-8445.

(8) Fischer, P.; Ghosh, A. Magnetically actuated propulsion at low Reynolds numbers: Towards nanoscale control. Nanoscale 2011, 3, 557-563.

(9) Agarwal, A.; Hess, H. Molecular motors as components of future medical devices and engineered materials. J. Nanotechnol. Eng. Med. 2009, 1, 011005.

(10) Wang, J.; Manesh, K. M. Motion control at the nanoscale. Small 2010, 6, 338-345.

(11) Hess, H.; Jaeger, L. Nanobiotechnology. Curr. Opin. Biotechnol. 2010, 21, 373-375. 
(12) Qin, L. D.; Banholzer, M. J.; Xu, X. Y.; Huang, L.; Mirkin, C. A. Rational design and synthesis of catalytically driven nanorotors. J. Am. Chem. Soc. 2007, 129, 14870-14871.

(13) Nelson, B. J.; Kaliakatsos, I. K.; Abbott, J. J. Microrobots for minimally invasive medicine. Ann. Rev. Biomed. Eng. 2010, 12, 55-85.

(14) Solovev, A. A.; Xi, W.; Gracias, D. H.; Harazim, S. M.; Deneke, C.; Sanchez, S.; Schmidt, O. G. Self-propelled nanotools. ACS Nano 2012, 6, 1751-1756.

(15) Sanchez, S.; Pumera, M. Nanorobots: The ultimate wireless selfpropelled sensing and actuating devices. Chem. Asian J. 2009, 4, 14021410.

(16) Wang, J.; Gao, W. Nano/microscale motors: Biomedical opportunities and challenges. ACS Nano 2012, 6, 5745-5751.

(17) Wang, W.; Castro, L. A.; Hoyos, M.; Mallouk, T. E. Autonomous motion of metallic microrods propelled by ultrasound. ACS Nano 2012, 6, 6122-6132.

(18) Kagan, D.; Benchimol, M. J.; Claussen, J. C.; Chuluun-Erdene, E.; Esener, S.; Wang, J. Acoustic droplet vaporization and propulsion of perfluorocarbon-loaded microbullets for targeted tissue penetration and deformation. Angew. Chem., Int. Ed. 2012, 51, 7519-7522.

(19) Barnett, S. B.; Ter Haar, G. R.; Ziskin, M. C.; Rott, H.-D.; Duck, F. A.; Maeda, K. International recommendations and guidelines for the safe use of diagnostic ultrasound in medicine. Ultrasound Med. Biol. 2000, 26, 355-366.

(20) Wang, W. Understanding the propulsion and assembly of autonomous nano- and micromotors powered by chemical gradients and ultrasound. Ph.D. Thesis, The Pennsylvania State University, University Park, PA, 2013.

(21) Bruus, H. Acoustofluidics 7: The acoustic radiation force on small particles. Lab Chip 2012, 12, 1578-1586.

(22) Lenshof, A.; Magnusson, C.; Laurell, T. Acoustofluidics 8: Applications of acoustophoresis in continuous flow microsystems. Lab Chip 2012, 12, 1210-1223.

(23) Friend, J.; Yeo, L. Y. Microscale acoustofluidics: Microfluidics driven via acoustics and ultrasonics. Rev. Mod. Phys. 2011, 83, 647704.

(24) Ding, X. Y.; Lin, S. C. S.; Kiraly, B.; Yue, H. J.; Li, S. X.; Chiang, I. K.; Shi, J. J.; Benkovic, S. J.; Huang, T. J. On-chip manipulation of single microparticles, cells, and organisms using surface acoustic waves. Proc. Natl. Acad. Sci. U. S. A. 2012, 109, 11105-11109.

(25) Shi, J.; Ahmed, D.; Mao, X.; Lin, S.-C. S.; Lawit, A.; Huang, T. J. Acoustic tweezers: patterning cells and microparticles using standing surface acoustic waves (SSAW). Lab Chip 2009, 9, 2890-2895.

(26) Chen, Y.; Ding, X.; Steven Lin, S.-C.; Yang, S.; Huang, P.-H.; Nama, N.; Zhao, Y.; Nawaz, A. A.; Guo, F.; Wang, W.; Gu, Y.; Mallouk, T. E.; Huang, T. J. Tunable nanowire patterning using standing surface acoustic waves. ACS Nano 2013, 7, 3306-3314.

(27) Kline, T. R.; Paxton, W. F.; Mallouk, T. E.; Sen, A. Catalytic nanomotors: Remote-controlled autonomous movement of striped metallic nanorods. Angew. Chem., Int. Ed. 2005, 44, 744-746.

(28) Solovev, A. A.; Sanchez, S.; Pumera, M.; Mei, Y. F.; Schmidt, O. G. Magnetic control of tubular catalytic microbots for the transport, assembly, and delivery of micro-objects. Adv. Funct. Mater. 2010, 20, 2430-2435.

(29) Garcia-Gradilla, V.; Orozco, J.; Sattayasamitsathit, S.; Soto, F.; Kuralay, F.; Pourazary, A.; Katzenberg, A.; Gao, W.; Shen, Y.; Wang, J. Fucntionalized ultrasound-propelled magnetically guided nanomotors: Toward practical biomedical applications. ACS Nano 2013, 7, 92329240.

(30) Love, J. C.; Urbach, A. R.; Prentiss, M. G.; Whitesides, G. M. Three-dimensional self-assembly of metallic rods with submicron diameters using magnetic interactions. J. Am. Chem. Soc. 2003, 125, 12696-12697.

(31) Happel, J.; Brenner, H. Low Reynolds Number Hydrodynamics; Prentice Hall: Englewood Cliffs, NJ, 1965; p 229.

(32) Ui, T. J.; Hussey, R. G. Stokes drag on a cylinder in axial motion. Phys. Fluids 1984, 27, 787-795.

(33) Paxton, W. F.; Kistler, K. C.; Olmeda, C. C.; Sen, A.; St. Angelo, S. K.; Cao, Y.; Mallouk, T. E.; Lammert, P.; Crespi, V. H. Autonomous movement of striped nanorods. J. Am. Chem. Soc. 2004, 126, 1342413431.

(34) Mino, G.; Mallouk, T. E.; Darnige, T.; Hoyos, M.; Dauchet, J.; Dunstan, J.; Soto, R.; Wang, Y.; Rousselet, A.; Clement, E. Enhanced diffusion due to active swimmers at a solid surface. Phys. Rev. Lett. 2011, 106, 048102/1-048102/4.

(35) Kline, T. R.; Tian, M.; Wang, J.; Sen, A.; Chan, M. W. H.; Mallouk, T. E. Template-grown metal nanowires. Inorg. Chem. 2006, $45,7555-7565$

(36) Wei, M. S.; Chou, S. Y. Size effects on switching field of isolated and interactive arrays of nanoscale single-domain $\mathrm{Ni}$ bars fabricated using electron beam nanolithography. J. Appl. Phys. 1994, 76, 66796681. 